\title{
Discrete-Phase Constant Envelope Precoding for Massive MIMO Systems
}

\author{
Mohammad Kazemi, Student Member, IEEE, Hassan Aghaeinia, and Tolga M. Duman, Fellow, IEEE
}

\begin{abstract}
We consider downlink of a multiuser massive multiple-input multiple-output (MIMO) system and focus on reducing the hardware costs by using a single common power amplifier and separate phase shifters (PSs) for antenna frontends. In the previous literature, the use of analog PSs in this setup has been considered. Here, we study the use of practical digital PSs, which only support a limited set of discrete phases. Considering the sum of interference powers as a metric, we formulate the corresponding nonlinear discrete optimization problem and solve for the phases to be used during transmission. We devise a low-complexity algorithm, which employs a trellis structure providing suboptimal, but efficient and effective solutions. We demonstrate via examples that the proposed solutions have comparable performance to conventional analog PS-based algorithms. Furthermore, we prove that by utilizing discretephase constant envelope precoding, the interference can be made arbitrarily small by increasing the number of antennas. Therefore, the asymptotic gains promised by massive MIMO systems are preserved. We also obtain closed-form expressions for the rate loss due to errors in the phase and amplitude of the PSs, for both low and high SNR regimes.
\end{abstract}

Index Terms-Constant envelope precoding, massive MIMO, multiuser MIMO, digital phase shifter, discrete optimization.

\section{INTRODUCTION}

$\mathbf{T}$ HE seminal work of [1] in 2010 has sparked a great interest in utilizing a large number of antennas at the base station (BS), a.k.a. massive multiple-input multiple-output (m-MIMO), in recent years. Numerous advantages of m-MIMO have made it one of the key technology enablers for the next generation of wireless communication systems [3], [17]. One of the main advantages of m-MIMO is that as the number of BS antennas goes to infinity, the performance of linear precoders approaches to that of optimal nonlinear ones [2], [18]. On the other hand, to implement the usual precoding algorithms, each antenna element needs a radio frequency (RF) power amplifier and a phase shifter (PS).

Manuscript received August 17, 2016; revised December 10, 2016 and January 23, 2017; accepted February 1, 2017. Date of publication February 13, 2017; date of current version May 13, 2017. This paper was presented in part at the 2016 Information Theory and Applications Workshop, La Jolla, CA. The work of T. M. Duman was supported in part by the EC Marie Curie Career Integration under Grant PCIG12-GA-2012-334213. The associate editor coordinating the review of this paper and approving it for publication was X. Gao.

M. Kazemi and H. Aghaeinia are with the Department of Electrical Engineering, Amirkabir University of Technology, Tehran 15875-4413, Iran (e-mail: mohammadkaazemi@aut.ac.ir; aghaeini@aut.ac.ir).

T. M. Duman is with the Department of Electrical and Electronics Engineering, Bilkent University, Ankara 06800, Turkey (e-mail: duman@ee.bilkent.edu.tr).

Color versions of one or more of the figures in this paper are available online at http://ieeexplore.ieee.org.

Digital Object Identifier 10.1109/TCOMM.2017.2667663
Therefore, by increasing the number of antennas, the number of RF elements, and hence the implementation cost, increases tremendously. One way to reduce this cost is to deploy a single common RF power amplifier for all antenna elements and a separate RF PS for each one, leading to antenna outputs with equal amplitudes but different phases. The resulting techniques are called constant envelope precoding (CEP) which were first considered in the context of m-MIMO in [4].

In CEP, since antenna elements have the same output amplitude and use a single common power amplifier, a highly power efficient nonlinear amplifier can be used instead of a linear one [4]. Typically, a nonlinear power amplifier can be about 4 to 6 times more power efficient [5]. Therefore, with CEP, higher power efficiency and lower cost can outweigh the slight performance degradation, especially in the m-MIMO regime.

In [6], considering constant envelope (CE) transmission, the authors derive achievable rates of a Gaussian broadcast channel for the special case of a single user. They extend their work to the case of multiuser precoding in [4]. They obtain the desired antenna phases by solving a nonlinear nonconvex continuous optimization problem with sum of squares of interuser interference terms as the objective function to be minimized. They prove that the performance of the resulting CEP algorithm is comparable to that of precoding under an average only total transmit power constraint (APC) in the mMIMO regime. To reduce the complexity, they also provide an alternating minimization (AM) method by taking all the phases but one to be a constant in each sub-iteration. That is, in each iteration, they solve $N$ one-dimensional problems instead of an $\mathrm{N}$-dimensional one. The authors extend their work to frequency selective channels in [7].

In [8], cross-entropy optimization (CEO) method is used to solve the CEP problem, and it is shown that the resulting performance is less sensitive to the selection of the initial phase guesses. In order to reduce the out-of-band emissions in the common nonlinear power amplifier, in [9], the authors present a CEP with small phase variations by limiting the difference of the phase angles transmitted in consecutive channel uses. They show that, by achieving low phase variations, the performance degrades by $3 \mathrm{~dB}$ with every doubling of the number of BS antennas.

Only analog PSs, which can support a continuous range of phase shifts, have been considered in the existing literature for CEP. However, in practice, due to advantages like increased immunity to noise on their voltage control lines, more uniform unit-to-unit performance and having flat phase over a wider 
bandwidth, mostly digital PSs are used [10]. Digital PSs provide a discrete set of phase states which are controlled by a string of binary digits; the higher the number of supported phase states, the higher the price. For instance, a 2-bit digital PS supports only 4 phase states, namely, 0, 90, 180 and 270 degrees [10].

In this paper, we show that the CEP design problem can be formulated as a nonlinear discrete optimization problem considering practical digital PSs. In general, discrete optimization problems are NP-hard, so they are usually solved using some suboptimal algorithms which usually have exponential time complexity [11]. Even in well-conditioned problems, when dimensions of the problem grow, as in the case of m-MIMO systems, their solution time is nontrivial and in most cases impractical. Therefore, innovative approaches are necessary to perform precoding in an efficient manner.

The contributions of this paper are fourfold: 1) considering digital PSs, the CEP design problem is formulated as a nonlinear discrete optimization problem, and then, using a trellis structure, a low-complexity solution is obtained, 2) a robust version of the proposed algorithm against channel estimation errors is derived, 3) it is shown that using CEP with discrete phases, interference powers at the users can be made arbitrarily small by taking the number of BS antennas to be sufficiently large, 4) the effects of phase and amplitude errors in practical PSs on the performance of CEP algorithms are analyzed.

Our main approach is as follows. We reformulate the interference power objective function as a sum of real terms. Then, by appropriately defining trellis states, we solve the corresponding nonlinear discrete optimization problem using a trellis structure in a manner similar to the Viterbi algorithm. Our proposed algorithm is compared with the technique of nonlinear optimization by mesh adaptive direct search (NOMAD) which has almost the same performance as other conventional discrete optimization techniques but it is much faster [13]. We demonstrate that the proposed approach has three main advantages. First, it is a few orders of magnitude less complex (hence much faster). Secondly, its performance is independent of the initial starting point. Thirdly, contrary to the conventional algorithms, its computational complexity is independent of factors such as input data or channel coefficients. We also demonstrate via extensive numerical results that the newly proposed algorithm has comparable or even better performance than NOMAD based solutions, especially in the m-MIMO regime.

We also provide some important analytical results. We prove that, similar to the case of continuous-phase CEP in [4], the user interference is guaranteed to be arbitrarily small in discrete-phase CEP by increasing the number of BS antennas. Closed-form expressions are also provided for the rate loss due to errors in phase and amplitude of the PSs for both low and high signal-to-noise ratio (SNR) regimes. The results show that by increasing the number of antennas, these effects do not fade away.

The rest of the paper is organized as follows. In Sections II and III, the system model and main problem formulation are introduced, respectively. In Section IV, the proposed trellis structure is presented and it is employed

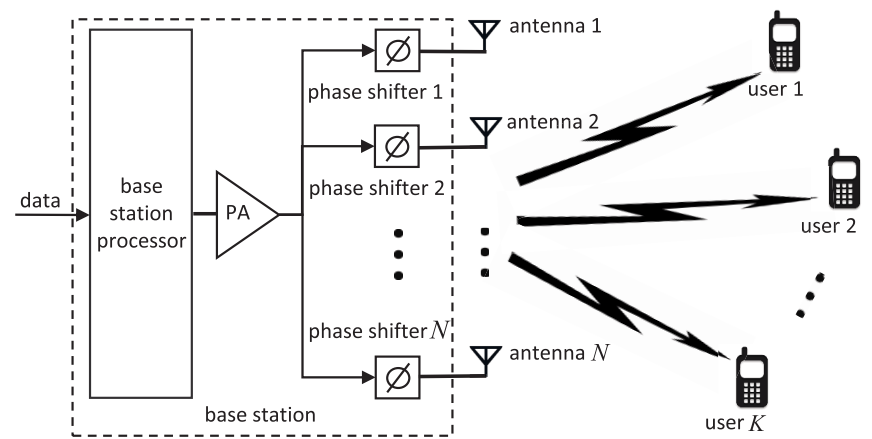

Fig. 1. System model showing downlink of a multiuser MIMO with the BS utilizing a single common power amplifier (PA) and separate PSs.

to solve the corresponding interference power minimization problem. The effects of number of BS antennas on the user interference, and phase and amplitude errors on the data rates are analyzed in Sections V and VI, respectively. Numerical examples are presented in Section VII. Finally, the paper is concluded in Section VIII.

\section{SYSTEM MODEL}

We consider downlink transmission in a m-MIMO system and assume that there is no cooperation among users. The system model consists of $K$ single antenna users and a BS with $N$ antennas. We assume that the BS knows the channel vector to each user. In our set-up, the BS utilizes a single common RF power amplifier for all the antennas and a separate RF PS for each one with the objective of CEP. The system model is illustrated in Fig. 1.

The received signal at the $k$ th user, $y_{k}$, can be written as

$$
y_{k}=\sqrt{\frac{P_{T}}{N}} \mathbf{h}_{k} \mathbf{q}+w_{k},
$$

where $\mathbf{q} \triangleq\left[e^{j \theta_{1}}, e^{j \theta_{2}}, \cdots, e^{j \theta_{N}}\right]^{\mathrm{T}}$ is the vector of CE signals transmitted from the BS, and $\theta_{i}$ is the output phase of the $i$ th antenna. In (1), $w_{k}$ is a zero-mean complex circularly symmetric Gaussian noise with variance $\sigma_{w}^{2}, \mathbf{h}_{k}$ is the channel vector to the $k$ th user ( $k$ th row of the $K \times N$ channel matrix $\mathbf{H}$ ), and $P_{T}$ is the total transmission power. We do not assume any specific fading channel model. While we provide numerical examples for Rayleigh fading channels, the proposed algorithm for CEP applies for any channel distribution, and the theoretical findings apply under very general conditions on the channel statistics specified in Sections V and VI.

\section{PROBlem Formulation}

Denoting the desired symbol for the $k$ th user by $x_{k}$, (1) can be divided into desired and interference parts as

$$
y_{k}=\underbrace{\sqrt{E_{k}} x_{k}}_{\text {desired }}+\underbrace{\left(\sqrt{\frac{P_{T}}{N}} \mathbf{h}_{k} \mathbf{q}-\sqrt{E_{k}} x_{k}\right)}_{\text {interference }}+w_{k},
$$

where $E_{k}$ denotes the symbol energy of the $k$ th user. Therefore, by considering the sum of interference powers at the users 
as the main criterion, the desired phases can be obtained as

$$
\begin{aligned}
& \widehat{\mathbf{q}}=\underset{\mathbf{q}}{\arg \min } \sum_{k=1}^{K}\left|\sqrt{\frac{P_{T}}{N}} \mathbf{h}_{k} \mathbf{q}-\sqrt{E_{k}} x_{k}\right|^{2}, \\
& \text { s.t. }\left|q_{n}\right|=1, \quad n=1,2, \cdots, N,
\end{aligned}
$$

which is a nonlinear nonconvex optimization problem.

As can be seen in (3), a challenge in CEP is, in general, that the corresponding optimization problem is a function of both the channel coefficients and the user data, hence the precoding coefficients are functions of them as well. Therefore, even when the channel does not change, the optimization should be performed in each data transmission interval.

All the previous CEP related works (e.g., [4], [7]-[9]) have considered the use of analog PSs, and have solved the optimization problem (3) by using such techniques as AM [4] and CEO [8]. However, as mentioned earlier, digital PSs may be more preferable in practice due to factors such as increased immunity to noise on their voltage control lines [10]. Assuming digital PSs which support a discrete set of phase states, (3) turns into a nonlinear discrete optimization problem, namely,

$$
\begin{aligned}
& \widehat{\mathbf{q}}=\underset{\mathbf{q}}{\arg \min } \sum_{k=1}^{K}\left|\sqrt{\frac{P_{T}}{N}} \mathbf{h}_{k} \mathbf{q}-\sqrt{E_{k}} x_{k}\right|^{2}, \\
& \text { s.t. } q_{n} \in Q_{M}, \quad n=1,2, \cdots, N,
\end{aligned}
$$

where $Q_{M}$ is a set of $M$ unit amplitude symbols (e.g., phase shift keying (PSK) symbols).

Taking $q_{n}=\exp \left(\frac{2 \pi m_{n}}{M}\right), m_{n} \in\{1, \cdots, M\}$, (4) turns into a nonlinear integer optimization problem and it can be solved by conventional integer programming methods such as branch-and-cut [16] and NOMAD. However, the worstcase complexity of such discrete (or integer) programming methods is exponential, and when the problem dimensions are large, their computational complexity is nontrivial, and in many cases, they become impractical even in well-conditioned problems [11]. Hence, they are not suitable options for the $\mathrm{m}$ MIMO scenario in which $N$ and consequently the problem dimension is very large.

To tackle this problem, we first expand the squares in the objective function of (4) and then take the summation over $k$. By ignoring the terms independent of $\mathbf{q}$, we have

$$
\begin{aligned}
& \widehat{\mathbf{q}}=\underset{\mathbf{q}}{\arg \min } \frac{P_{T}}{N} \sum_{j=1}^{N} \sum_{i=1}^{N} q_{j}^{*} \mathbf{G}_{j, i} q_{i}-2 \sqrt{\frac{P_{T}}{N}} \sum_{j=1}^{N} \operatorname{Re}\left\{\mathbf{x}^{\mathrm{H}} \mathbf{E H}_{j} q_{j}\right\} \\
& \text { s.t. } q_{n} \in Q_{M}, \quad n=1,2, \cdots, N,
\end{aligned}
$$

where $\mathbf{H}_{j}$ is the $j$ th column of the channel matrix $\mathbf{H}, \mathbf{G} \stackrel{\Delta}{=}$ $\mathbf{H}^{\mathrm{H}} \mathbf{H}, \mathbf{x}$ is the $K \times 1$ vector of desired users' symbols, and $\mathbf{E}$ is a $K \times K$ diagonal matrix whose $k$ th diagonal element is $\sqrt{E}_{k}$. Since $\mathbf{G}$ is a Hermitian matrix, we can write $\sum_{j=1}^{N} \sum_{i=1}^{N} q_{j}^{*} \mathbf{G}_{j, i} q_{i}=\sum_{j=1}^{N} \mathbf{G}_{j, j}\left|q_{j}\right|^{2}+2 \sum_{j=1}^{N} \sum_{i=1}^{j-1} \operatorname{Re}\left\{q_{j}^{*} \mathbf{G}_{j, i} q_{i}\right\}$. Substituting this into (5) and knowing that $\left|q_{j}\right|=1$, the final

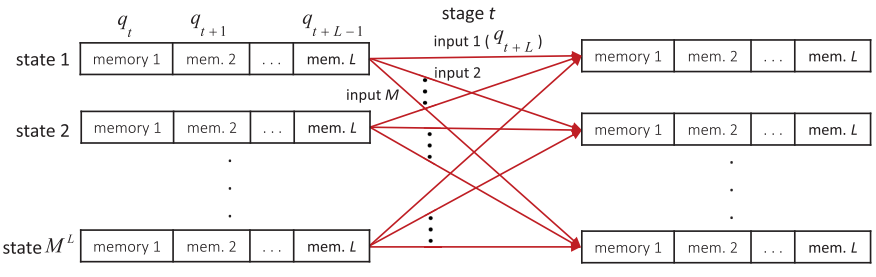

Fig. 2. Trellis structure of TB-CEP.

form of the discrete optimization problem becomes

$$
\begin{aligned}
& \widehat{\mathbf{q}}=\underset{\mathbf{q}}{\arg \min } \sum_{j=1}^{N} \operatorname{Re}\left\{\sqrt{\frac{P_{T}}{N}} \sum_{i=1}^{j-1} q_{j}^{*} \mathbf{G}_{j, i} q_{i}-\mathbf{x}^{\mathrm{H}} \mathbf{E} \mathbf{H}_{j} q_{j}\right\} \\
& \text { s.t. } q_{n} \in Q_{M}, \quad n=1,2, \cdots, N .
\end{aligned}
$$

In this form, the objective function is transformed into sum of $N$ real terms in which the $j$ th term is only a function of the first $j$ variables. In the next section, we propose a trellis based optimization algorithm which exploits this property to provide a suboptimal but efficient and effective method.

\section{Discrete-Phase Constant Envelope Precoding}

We reformulated the discrete-phase CEP problem in such a way that sum of $N$ real terms in which the $j$ th term is only a function of first $j$ variables is to be optimized. We now propose a low-complexity algorithm which exploits this special structure, that is, by appropriately defining trellis states, we introduce a low-complexity trellis-based solution to the main discrete optimization problem in (6). We analyze the computational complexity of the proposed algorithm in the remaining two subsections.

\section{A. Proposed Trellis-Based CEP}

Since the objective function in (6) is the sum of $N$ real terms in which the $j$ th term is only a function of the first $j$ variables, the problem can be solved using a multistage approach. A simple way is the greedy approach. That is, at the $j$ th stage, we can select the symbol in the set $Q_{M}$ (corresponding to the phases for the PSs) which minimizes the $j$ th term of the objective function of (6) as the $j$ th antenna output. However, we make the observation that if we keep the most likely set of variables at each stage instead of only one variable, and make the overall decision at the end of the algorithm's run, we can obtain improved results. We can accomplish this by employing a trellis structure much like what the Viterbi algorithm utilizes in decoding or channel equalization. We call the resulting algorithm trellis-based CEP (TB-CEP).

Define the previous $L q_{i}$ variables as the memory, each taking on $M$ possible values. Hence, we have a trellis with $M^{L}$ states, each having $M$ outgoing branches with labels chosen from $Q_{M}$ (e.g., set of $M$-PSK symbols) as illustrated in Fig. 2.

The proposed algorithm (TB-CEP) works as follows. At the first step, the initial $L$ variables are taken as initial memory values and their $M^{L}$ possible combinations form the trellis states. Initial metrics are determined by putting the values of each state in the first $L$ terms of the objective function of (6). At the $t$ th stage, the branch labels correspond to the $(t+L)$ th 


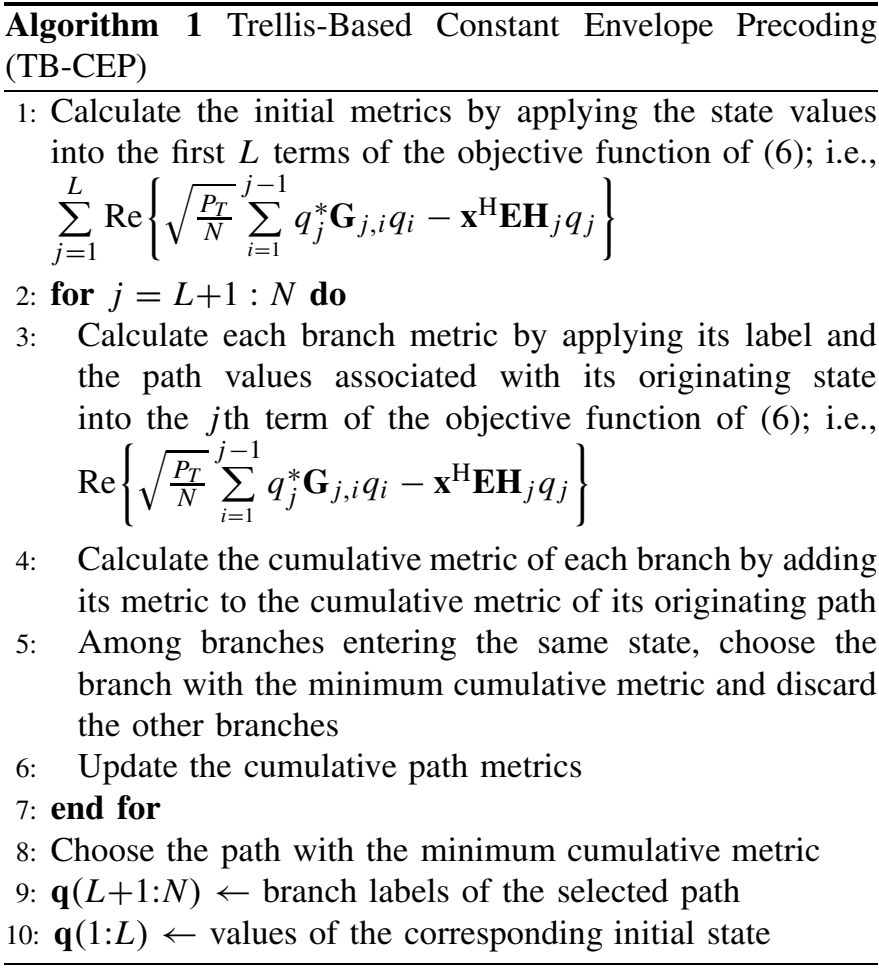

variable $\left(q_{t+L}\right)$, and the branch metric is the $(t+L)$ th term of the objective function of (6). At each stage, the cumulative metric of branches are calculated by adding the branch metrics to the cumulative metric of their originating paths. Then, among the branches entering the same state, the branch with the least cumulative metric is kept and the others are discarded. The algorithm is terminated after $N-L$ stages and the path with the minimum cumulative metric is selected as the final decision. The labels on the selected path give the latter $N-L$ variables, and the initial state associated with the selected path gives the first $L$ variables. The procedure is summarized in Algorithm 1. It should be noted that the proposed algorithm is not iterative and solves the optimization problem in a single run.

\section{B. Computational Complexity Analysis}

In our proposed algorithm, we have $N-L$ stages and $M^{L}$ states, with $M$ branches entering each state. Therefore, there are a total of $(N-L) M^{L+1}$ comparisons. Since the terms of (6) are used as the branch metrics, the numbers of additions and multiplications are both $O\left(N^{2} K M^{L+1}\right)$ for each channel use. In other words, the complexity is polynomial in the number of BS antennas which is significantly better than the alternatives which have exponential complexity [11]. We further note that the complexity of the proposed algorithm is comparable to that of continuous-phase CEP and also to conventional linear precoders (without the CE constraint). For example, the numbers of additions and multiplications in zero forcing $(\mathrm{ZF})$ precoding are $O\left(N K^{2}\right)$ for carrying out the pseudo-inverse in each coherence time interval of the channel, and $O(N K)$ for matrix vector product in each channel use [4]. The continuous-phase CEP has a complexity of $O\left(N K N_{\text {iter }}\right)$ in each channel use, where $N_{\text {iter }}$ is the number of iterations used in the optimization process [4].

Our numerical results in Section VII show that the proposed algorithm is a few orders of magnitude faster than the conventional discrete programming algorithms such as NOMAD in the m-MIMO scenario. Furthermore, unlike the conventional discrete programming algorithms, the complexity and running time of the proposed algorithm are fixed and independent of factors like user data and channel coefficients. Finally, the proposed algorithm does not need an initial point, on the contrary, the performance and speed of the conventional algorithms heavily depend on the choice of initialization.

\section{Robust CEP}

In practice, the channel coefficients are estimated with some error. Hence, we need to focus on the effects of estimation errors on the performance of the proposed algorithm. Let the estimated channel matrix be $\hat{\mathbf{H}} \triangleq \mathbf{H}+\tilde{\mathbf{H}}$, where $\tilde{\mathbf{H}}$ denotes the error values. Assuming that $\tilde{\mathbf{H}}$ has zero mean entries and $\mathbf{C} \triangleq \mathrm{E}\left[\tilde{\mathbf{H}}^{\mathrm{H}} \tilde{\mathbf{H}}\right]$, using (3), a robust CEP problem can be posed as follows [19]:

$$
\begin{aligned}
& \widehat{\mathbf{q}}=\underset{\mathbf{q}}{\arg \min } \sum_{k=1}^{K}\left|\sqrt{\frac{P_{T}}{N}} \hat{\mathbf{h}}_{k} \mathbf{q}-\sqrt{E_{k}} x_{k}\right|^{2}+\frac{P_{T}}{N}\left\|\mathbf{C}^{\frac{1}{2}} \mathbf{q}\right\|_{2}^{2}, \\
& \text { s.t. }\left|q_{n}\right|=1, \quad n=1,2, \cdots, N .
\end{aligned}
$$

Since $\mathbf{C}$ is a Hermitian matrix and $\left|q_{n}\right|=1$, we have $\left\|\mathbf{C}^{\frac{1}{2}} \mathbf{q}\right\|_{2}^{2}=$ $\sum_{j=1}^{N} \sum_{i=1}^{N} q_{j}^{*} \mathbf{C}_{j, i} q_{i}=\sum_{j=1}^{N} \mathbf{C}_{j, j}+2 \sum_{j=1}^{N} \sum_{i=1}^{j-1} \operatorname{Re}\left\{q_{j}^{*} \mathbf{C}_{j, i} q_{i}\right\}$. Therefore, in the same manner as Section III, using (7), the robust disretephase CEP problem becomes:

$$
\begin{aligned}
& \widehat{\mathbf{q}}=\underset{\mathbf{q}}{\arg \min } \sum_{j=1}^{N} \operatorname{Re}\left\{\sqrt{\frac{P_{T}}{N}} \sum_{i=1}^{j-1} q_{j}^{*} \mathbf{B}_{j, i} q_{i}-\mathbf{x}^{\mathrm{H}} \mathbf{E} \hat{\mathbf{H}}_{j} q_{j}\right\} \\
& \text { s.t. } q_{n} \in Q_{M}, \quad n=1,2, \cdots, N,
\end{aligned}
$$

where $\mathbf{B} \stackrel{\Delta}{=} \mathrm{E}\left[\hat{\mathbf{H}}^{\mathrm{H}} \hat{\mathbf{H}}\right]+\mathbf{C}$.

Note that by substituting $\mathbf{G}$ with $\mathbf{B}$ in the non-robust discrete-phase CEP problem (6), we obtain the above robust formulation. Therefore, the robust discrete-phase CEP problem can be solved using the proposed TB-CEP algorithm (summarized in Algorithm 1) by substituting $\mathbf{G}$ with $\mathbf{B}$. We call the resulting algorithm the robust TB-CEP. For a more detailed analysis and management of channel uncertainties in MIMO systems, see [20] and the references therein.

\section{ANALYSiS OF INTERFERENCE With INCREASING NUMBER OF BS ANTENNAS}

In this section, we show that the interference terms at the receivers in CEP with discrete phases can be made arbitrarily small by taking $N$ sufficiently large. This is proved to hold for the case of continuous-phase CEP in [4], hence we only state and prove the result as an extension to the case of digital PSs in Theorem 1. The general idea of the proof is as follows. Not considering noise and taking the channel 
vectors and the transmission power as constants, the number of possible received signal vectors at the users is equal to $M^{N}$, each one related to a specific set of antenna output phases. So by increasing the number of BS antennas, the number of possible received signal vectors increases. That is, since the transmission power is kept constant, the space of the received signal vectors becomes denser. As a result, under mild conditions on the channel gains, the probability that there exists a received signal vector (or equivalently, a set of antenna output phases), whose distance to the vector of desired symbols is smaller than a certain value, increases. This probability can be shown to be nonzero, indicating that there is a solution with the desired precoding properties.

Theorem 1: Fix the number of users $K$. Considering the random channel coefficients matrices, $\left\{\mathbf{H}_{N}\right\}_{N=K}^{\infty}$, which satisfy $\lim _{N \rightarrow \infty} \frac{\mathrm{E}\left[h_{k, i} h_{r, i} h_{s, i}^{*} h_{t, i}^{*}\right]}{N}=0, \forall k, r, s, t \in 1,2, \ldots, K$, for any data vector $\mathbf{x}$ chosen from a fixed finite alphabet $\chi$ and any $\Delta>0$, there exists an integer $N^{\prime}\left(\left\{\mathbf{H}_{N}\right\}, \chi, \Delta\right)$ such that with $N \geq N^{\prime}\left(\left\{\mathbf{H}_{N}\right\}, \chi, \Delta\right)$, there exists a phase vector $\theta_{N}^{x}(\Delta)=$ $\left[\theta_{1}^{x}(\Delta), \ldots, \theta_{N}^{x}(\Delta)\right]^{\mathrm{T}}$ chosen from a set of discrete phases which when transmitted results in the multiuser interference energy at each user being upper bounded by $2 \Delta^{2}$. In other words, the user interference in discrete-phase CEP can be guaranteed to be arbitrarily small as $N \rightarrow \infty$.

Proof: The continuous-phase case of Theorem 1 is proved in [4]. The proof of the discrete case follows similar steps with some necessary modifications. The proof is based on showing that the vector of noiseless received signals at the users is asymptotically jointly normal which, in [4], is only proved to hold for the case of continuous phases. Since the proof in [4], except the part of joint normality, is independent of the phases being continuous or discrete, in order to avoid repetition, here we only show that joint normality also holds for the case of discrete phases, and refer the reader to [4] for the rest.

Result 1: Let $\left\{X_{n}\right\}$ be a sequence of independent random variables $(\mathrm{RV})$. Let $\mathrm{E}\left[X_{n}\right]=\mu_{n}, \mathrm{E}\left[\left(X_{n}-\mu_{n}\right)^{2}\right]=\sigma_{n}^{2}$, and for some fixed $\xi>0, \mathrm{E}\left[\left|X_{n}-\mu_{n}\right|^{2+\xi}\right]=\beta_{n}$ exists for all $n$. Furthermore let $B_{n} \triangleq\left(\sum_{i=1}^{n} \beta_{i}\right)^{\frac{1}{2+\xi}}$ and $C_{n} \triangleq\left(\sum_{i=1}^{n} \sigma_{i}^{2}\right)^{\frac{1}{2}}$. Then if $\lim _{n \rightarrow \infty} \frac{B_{n}}{C_{n}}=0$ (to ensure that no one variable $X_{n}$ dominates the summation), the cumulative distribution function (c.d.f.) of $Y_{n}=\frac{\sum_{i=1}^{n}\left(X_{i}-\mu_{i}\right)}{C_{n}}$ converges in the limit to the c.d.f. of a real Gaussian RV with mean zero and unit variance [14].

Result 2: If every linear combination of a set of RV's has normal distribution, then they are jointly normal [12].

To complete the proof of Theorem 1, first using Result 1, we show that every linear combination of the noiseless received signals at the users, $z_{k}=\sqrt{\frac{P_{T}}{N}} \mathbf{h}_{k} \mathbf{q}$, has asymptotically a normal distribution. Then, using Result 2, we conclude that the noiseless received signals at the users are jointly normally distributed. We define the linear combination of the noiseless received signals

$$
\zeta \triangleq \sum_{k=1}^{K} \lambda_{k} z_{k}=\sqrt{\frac{P_{T}}{N}} \sum_{k=1}^{K} \lambda_{k} \mathbf{h}_{k} \mathbf{q}=\sum_{i=1}^{N} \eta_{i},
$$

where $\eta_{i} \triangleq \sqrt{\frac{P_{T}}{N}} e^{j} \theta_{i} \sum_{k=1}^{K} \lambda_{k} h_{k, i}$ and $\lambda_{k}$ is an arbitrary coefficient. Since Theorem 1 is an existence theorem, only stating that such a discrete-phase precoder exists, we can assume the phase angles to be independent of each other, and hence $\eta_{i}$ 's become independent. Without loss of generality, we assume the output phases to be uniformly distributed, therefore for the mean and the variance of $\eta_{i}$, we have

$$
\begin{aligned}
& \mu_{i} \triangleq \mathrm{E}\left[\eta_{i}\right]=\sqrt{\frac{P_{T}}{N}} \mathrm{E}\left[e^{j \theta_{i}}\right] \sum_{k=1}^{K} \lambda_{k} h_{k, i}=0, \\
& \sigma_{i}^{2} \triangleq \mathrm{E}\left[\left|\eta_{i}-\mu_{i}\right|^{2}\right]=\mathrm{E}\left[\left|\eta_{i}\right|^{2}\right]=\frac{P_{T}}{N}\left|\sum_{k=1}^{K} \lambda_{k} h_{k, i}\right|^{2} .
\end{aligned}
$$

For $\xi=2$, we have

$$
\beta_{i}=\mathrm{E}\left[\left|\eta_{i}\right|^{4}\right]=\left(\frac{P_{T}}{N}\right)^{2}\left|\sum_{k=1}^{K} \lambda_{k} h_{k, i}\right|^{4},
$$

which exists for all $i$. Now, using (10) and (11), we calculate $B_{N}$ and $C_{N}$ as follows.

$B_{N}=\left(\frac{P_{T}^{2}}{N} \sum_{k=1}^{K} \sum_{r=1}^{K} \sum_{s=1}^{K} \sum_{t=1}^{K} \lambda_{k} \lambda_{r} \lambda_{s}^{*} \lambda_{t}^{*} \frac{1}{N} \sum_{i=1}^{N} h_{k, i} h_{r, i} h_{s, i}^{*} h_{t, i}^{*}\right)^{\frac{1}{4}}$,

$C_{N}=\left(P_{T} \sum_{k=1}^{K} \sum_{t=1}^{K} \lambda_{k} \lambda_{t}^{*} \frac{1}{N} \sum_{i=1}^{N} h_{k, i} h_{t, i}^{*}\right)^{\frac{1}{2}}$

Using the law of large numbers, the normalized summations of the product of channel coefficients in (13) and (13) asymptotically tend to their expected values for large $N$. Hence, we obtain

$B_{N} \rightarrow\left(\frac{P_{T}^{2}}{N} \sum_{k=1}^{K} \sum_{r=1}^{K} \sum_{s=1}^{K} \sum_{t=1}^{K} \lambda_{k} \lambda_{r} \lambda_{s}^{*} \lambda_{t}^{*} \mathrm{E}\left[h_{k, i} h_{r, i} h_{s, i}^{*} h_{t, i}^{*}\right]\right)^{\frac{1}{4}}$,

$C_{N} \rightarrow\left(P_{T}\left(\sum_{k=1}^{K} \sum_{\substack{t=1 \\ t \neq k}}^{K} \lambda_{k} \lambda_{t}^{*}\left|\mathrm{E}\left[h_{k, i}\right]\right|^{2}+\sum_{k=1}^{K}\left|\lambda_{k}\right|^{2} \mathrm{E}\left[\left|h_{k, i}\right|^{2}\right]\right)\right)^{\frac{1}{2}}$,

where $C_{N}$ is a nonzero constant independent of $N$ (ignoring the trivial case of all-zero $\lambda_{k}$ 's). Using (14) and (15), we have

$$
\begin{aligned}
\lim _{N \rightarrow \infty} \frac{B_{N}}{C_{N}} \\
=\frac{\left(P_{T}^{2} \sum_{k=1}^{K} \sum_{r=1}^{K} \sum_{s=1}^{K} \sum_{t=1}^{K} \lambda_{k} \lambda_{r} \lambda_{s}^{*} \lambda_{t}^{*} \lim _{N \rightarrow \infty} \frac{\mathrm{E}\left[h_{k, i} h_{r, i} h_{s, i}^{*} h_{t, i}^{*}\right]}{N}\right)^{\frac{1}{4}}}{\left(P_{T}\left(|\mu|^{2}\left(\left|\sum_{k=1}^{K} \lambda_{k}\right|^{2}-\sum_{k=1}^{K}\left|\lambda_{k}\right|^{2}\right)+\sigma^{2} \sum_{k=1}^{K}\left|\lambda_{k}\right|^{2}\right)\right)^{\frac{1}{2}}} \\
=0
\end{aligned}
$$

where we employed the assumption $\lim _{N \rightarrow \infty} \frac{\mathrm{E}\left[h_{k, i} h_{r, i} h_{s, i}^{*} h_{t, i}^{*}\right]}{N}=0$, $\forall k, r, s, t \in 1,2, \ldots, K$. Note that this is a very mild constraint 
satisfied with many interesting fading channel models including Rayleigh and Rician fading scenarios. Hence, we conclude that the Lyapunov CLT holds for the case of the discrete phases. Therefore, using Result 2, the noiseless received signals at the users are jointly normal, and the rest of the proof is the same as the continuous-phase case [4].

Theorem 1 basically says that by increasing $N$, the user interference fades away, and hence the users' interference links turn into noninterfering parallel links, increasing the user data rates. This fact reinforces our choice of user interference as the design criterion in the precoding design problem which is discussed in Sections III and IV. More importantly, Theorem 1 implies that restricting the phases of the antenna elements to a limited set has no effect on the performance of the system when $N$ is sufficiently large (as in the m-MIMO systems).

\section{Rate loss Due to Phase and Amplitude ERRORS OF PHASE SHIFTERS}

PSs are not ideal in practice. A nonideal PS changes the amplitude of the input signal, and also its output phase is not exactly the same as the set of predefined discrete phases. In a PS, these deviations from the ideal case are called phase and amplitude errors, respectively. In this section, we analyze the effects of such errors on the performance of CEP systems. To achieve this goal, closed-form expressions for rate loss due to PS phase and amplitude errors are obtained for both low and high SNR regimes.

The following is shown to be an achievable rate for the $k$ th user in CEP systems [4]

$$
R_{k}(\boldsymbol{\theta})=\log _{2}\left(\frac{E_{k}}{I_{k}(\boldsymbol{\theta})+\sigma_{w}^{2}}\right),
$$

where $\boldsymbol{\theta}=\left[\theta_{1}, \theta_{2}, \ldots, \theta_{N}\right]$ is the vector of BS output phases and $I_{k}(\boldsymbol{\theta}) \triangleq \mathrm{E}_{h, x}\left[\left|\sqrt{\frac{P_{T}}{N}} \sum_{i=1}^{N} h_{k, i} e^{j \theta_{i}}-\sqrt{E_{k}} x_{k}\right|^{2}\right]$ is the average interference power at the $k$ th user. Here, we employ this rate as a performance metric as well. We note that since PS errors occur after the precoder optimization, they do not affect the optimization itself, however, they impact the overall system performance.

Using (17), the following achievable rate can be obtained in the presence of phase and amplitude errors

$$
R_{k}(\boldsymbol{\theta}+\boldsymbol{\epsilon}, \boldsymbol{\alpha})=\log _{2}\left(\frac{E_{k}}{I_{k}(\boldsymbol{\theta}+\boldsymbol{\epsilon}, \boldsymbol{\alpha})+\sigma_{w}^{2}}\right),
$$

where $\boldsymbol{\epsilon}=\left[\epsilon_{1}, \epsilon_{2}, \ldots, \epsilon_{N}\right]$ and $\boldsymbol{\alpha}=\left[\alpha_{1}, \alpha_{2}, \ldots, \alpha_{N}\right]$ are the vectors of phase and amplitude errors, respectively, and $I_{k}(\boldsymbol{\theta}+\boldsymbol{\epsilon}, \boldsymbol{\alpha}) \triangleq \mathrm{E}_{h, x}\left[\left|\sqrt{\frac{P_{T}}{N}} \sum_{i=1}^{N} h_{k, i}\left(1+\alpha_{i}\right) e^{j\left(\theta_{i}+\varepsilon_{i}\right)}-\sqrt{E_{k}} x_{k}\right|^{2}\right]$ is the average interference power at the $k$ th user in the presence of phase and amplitude errors. We define the rate loss of the $k$ th user due to phase and amplitude errors as follows

$$
\Delta R_{k} \triangleq \mathrm{E}_{\varepsilon, \alpha}\left[R_{k}(\boldsymbol{\theta})-R_{k}(\boldsymbol{\theta}+\boldsymbol{\epsilon}, \boldsymbol{\alpha})\right] .
$$

Theorem 2: Let the PSs have zero mean amplitude and zero mean symmetric phase error vectors with covariance matrices of $\sigma_{\varepsilon}^{2} \mathbf{I}$ and $\sigma_{\alpha}^{2} \mathbf{I}$, respectively. Then, in a CEP with $K \ll N$, the rate loss expressions of the $k$ th user due to phase and amplitude errors are approximately equal to

$$
\begin{gathered}
\Delta R_{k}^{\text {lowSNR }} \approx \log _{2}(e) \frac{P_{T}}{\sigma_{w}^{2}}\left(\left(1+\rho_{k}\right)+\sigma_{\alpha}^{2}-\left(1+\rho_{k}\right) \varphi_{\mathrm{E}}^{2}(1)\right), \\
\Delta R_{k}^{\text {highSNR }} \approx \log _{2}\left(\frac{P_{T}}{\sigma_{w}^{2}}\left(\left(1+\rho_{k}\right)+\sigma_{\alpha}^{2}-\left(1+\rho_{k}\right) \varphi_{\mathrm{E}}^{2}(1)\right)\right),
\end{gathered}
$$

where $\varphi_{\mathrm{E}}(\cdot)$ denotes the characteristic function of the phase error and $\rho_{k} \triangleq \frac{E_{k}}{P_{T}}$. Note that the low SNR and high SNR regimes are defined as $\sigma_{w}^{2} \gg \mathrm{E}_{\varepsilon, \alpha}\left[I_{k}(\boldsymbol{\theta}+\boldsymbol{\epsilon}, \boldsymbol{\alpha})\right]$ and $\sigma_{w}^{2} \ll \mathrm{E}_{\varepsilon, \alpha}\left[I_{k}(\boldsymbol{\theta}+\boldsymbol{\epsilon}, \boldsymbol{\alpha})\right]$, respectively.

Proof: We make the following approximation: a good precoder minimizes the objective function well enough for $K \ll N$ to make the interference power at each user much smaller than the noise power, i.e., $I_{k}(\boldsymbol{\theta}) \ll \sigma_{w}^{2}$ (this is verified by our simulations of the CEP algorithms for large number of antennas). Therefore, the data rate (17) can be approximated as follows

$$
R_{k}(\boldsymbol{\theta}) \approx \log _{2}\left(\frac{E_{k}}{\sigma_{w}^{2}}\right) .
$$

First, we consider the case that phase and amplitude errors do not increase each user's interference power more than the noise power. That is, $\mathrm{E}_{\varepsilon, \alpha}\left[I_{k}(\boldsymbol{\theta}+\boldsymbol{\epsilon}, \boldsymbol{\alpha})\right] \ll \sigma_{w}^{2}$, or equivalently we operate in the low SNR regime. In this case, using first-order Taylor approximations $\log _{2}(1+\delta) \approx \delta \log _{2}(e)$ and $\frac{1}{1+\delta} \approx 1-\delta$ $(|\delta| \ll 1)$, and considering phase and amplitude errors, an approximation for the data rate (18) can be obtained as

$$
\begin{aligned}
R_{k}(\boldsymbol{\theta}+\boldsymbol{\epsilon}, \boldsymbol{\alpha}) & \approx \log _{2}\left(\frac{E_{k}}{\sigma_{w}^{2}}\left(1-\frac{I_{k}(\boldsymbol{\theta}+\boldsymbol{\epsilon}, \boldsymbol{\alpha})}{\sigma_{w}^{2}}\right)\right) \\
& =\log _{2}\left(\frac{E_{k}}{\sigma_{w}^{2}}\right)+\log _{2}\left(1-\frac{I_{k}(\boldsymbol{\theta}+\boldsymbol{\epsilon}, \boldsymbol{\alpha})}{\sigma_{w}^{2}}\right) \\
& \approx \log _{2}\left(\frac{E_{k}}{\sigma_{w}^{2}}\right)-\log _{2}(e) \frac{I_{k}(\boldsymbol{\theta}+\boldsymbol{\epsilon}, \boldsymbol{\alpha})}{\sigma_{w}^{2}} .
\end{aligned}
$$

Using (22) and (23), the approximate rate loss of the $k$ th user in the low SNR regime due to phase and amplitude errors is

$$
\Delta R_{k}^{\text {lowSNR }} \approx \frac{\log _{2}(e)}{\sigma_{w}^{2}} \mathrm{E}_{\varepsilon, \alpha}\left[I_{k}(\boldsymbol{\theta}+\boldsymbol{\epsilon}, \boldsymbol{\alpha})\right] .
$$

The expected value of the interference power in the presence of phase and amplitude errors is obtained in the Appendix as follows

$\mathrm{E}_{\varepsilon, \alpha}\left[I_{k}(\boldsymbol{\theta}+\boldsymbol{\epsilon}, \boldsymbol{\alpha})\right] \approx P_{T}\left(\left(1+\rho_{k}\right)+\sigma_{\alpha}^{2}-\left(1+\rho_{k}\right) \varphi_{\mathrm{E}}^{2}(1)\right)$.

Substituting (25) into (24) gives (20).

We next consider the case that phase and amplitude errors increase the user interference powers more than the noise power. That is, $\mathrm{E}_{\varepsilon, \alpha}\left[I_{k}(\boldsymbol{\theta}+\boldsymbol{\epsilon}, \boldsymbol{\alpha})\right] \gg \sigma_{w}^{2}$, or equivalently the high SNR regime. In this case, the data rate can be approximated in a similar manner to (23) as follows

$$
\begin{aligned}
R_{k}(\boldsymbol{\theta}+\boldsymbol{\epsilon}, \boldsymbol{\alpha}) & \approx \log _{2}\left(\frac{E_{k}}{I_{k}(\boldsymbol{\theta}+\boldsymbol{\epsilon}, \boldsymbol{\alpha})}\right)-\frac{\log _{2}(e) \sigma_{w}^{2}}{I_{k}(\boldsymbol{\theta}+\boldsymbol{\epsilon}, \boldsymbol{\alpha})} \\
& \approx \log _{2}\left(\frac{E_{k}}{\sigma_{w}^{2}}\right)+\log _{2}\left(\frac{\sigma_{w}^{2}}{I_{k}(\boldsymbol{\theta}+\boldsymbol{\epsilon}, \boldsymbol{\alpha})}\right) .
\end{aligned}
$$


Therefore, using (22) and (26), the approximate rate loss of the $k$ th user in the high SNR regime is

$$
\begin{aligned}
\Delta R_{k}^{\mathrm{highSNR}} & \approx \mathrm{E}_{\varepsilon, \alpha}\left[\log _{2}\left(\frac{I_{k}(\boldsymbol{\theta}+\boldsymbol{\epsilon}, \boldsymbol{\alpha})}{\sigma_{w}^{2}}\right)\right] \\
& \approx \log _{2}\left(\frac{\mathrm{E}_{\varepsilon, \alpha}\left[I_{k}(\boldsymbol{\theta}+\boldsymbol{\epsilon}, \boldsymbol{\alpha})\right]}{\sigma_{w}^{2}}\right) .
\end{aligned}
$$

Finally, (21) is obtained by substituting (25) into (27).

It should be noted that we do not consider the channel coefficients and precoding weights to be independent of each other in the above arguments. Theorem 2 implies that by increasing $N$, the effects of phase and amplitude errors in the PSs do not fade away. One reason is that these errors occur independently of the precoding optimization in the antenna front-ends and are not accounted for in the optimization process. Note also that the result obtained in Theorem 2 is for general CEP and not restricted to the discrete case.

Corollary 1: For the case of phase errors with sufficiently small variances, the rate loss expressions (20) and (21) can be further approximated as

$$
\begin{gathered}
\Delta R_{k}^{\text {lowSNR }} \approx \log _{2}(e) \frac{P_{T}}{\sigma_{w}^{2}}\left(\sigma_{\alpha}^{2}+\left(1+\rho_{k}\right) \sigma_{\epsilon}^{2}\right), \\
\Delta R_{k}^{\text {highSNR }} \approx \log _{2}\left(\frac{P_{T}}{\sigma_{w}^{2}}\left(\sigma_{\alpha}^{2}+\left(1+\rho_{k}\right) \sigma_{\epsilon}^{2}\right)\right) .
\end{gathered}
$$

Proof: Assuming the phase error to be sufficiently small and knowing that it is zero mean, the second order Taylor approximation of its characteristics function becomes

$$
\varphi_{\mathrm{E}}(t) \triangleq \mathrm{E}_{\varepsilon}\left[e^{j t \varepsilon}\right] \approx 1+j t \mathrm{E}_{\varepsilon}[\varepsilon]-\frac{1}{2} t^{2} \mathrm{E}_{\varepsilon}\left[\varepsilon^{2}\right]=1-\frac{1}{2} t^{2} \sigma_{\varepsilon}^{2} .
$$

Using (30) and knowing that $\sigma_{\varepsilon}^{2} \gg \sigma_{\varepsilon}^{4}$, the rate loss expressions (20) and (21) can be further simplified as stated in (28) and (29), respectively.

Corollary 2: Assuming sufficiently small error variances, the normalized rate loss (defined as $\Delta R_{k} / R_{k}(\theta)$ ) in the high SNR regime due to phase and amplitude errors of the PSs is

$$
\frac{\Delta R_{k}^{\text {highSNR }}}{R_{k}(\boldsymbol{\theta})} \approx 1+\frac{\log _{2}\left(\frac{1}{\rho_{k}} \sigma_{\alpha}^{2}+\left(\frac{1}{\rho_{k}}+1\right) \sigma_{\varepsilon}^{2}\right)}{\log _{2}\left(\frac{\rho_{k} \mathrm{P}_{\mathrm{T}}}{\sigma_{w}^{2}}\right)} .
$$

Proof: (31) is readily obtained using (22) and (29).

Corollary 2 shows that even the normalized rate loss due to phase and amplitude errors is independent of $N$ and it does not fade away as $N$ increases. According to Corollary 2, the normalized rate approaches one at very high transmit SNRs $\left(\mathrm{P}_{\mathrm{T}} / \sigma_{w}^{2}\right)$, which makes CEP algorithms impractical in that regime. However, our simulations show that, in the high (but still practical) SNR regime, the normalized rate loss is not too detrimental, and the proposed CEP solution remains effective.

\section{NUMERICAL EXAMPLES}

In this section, we first study the performance of the proposed TB-CEP algorithm in various set-ups and compare it with other alternatives. Then, we investigate its complexityperformance trade-off. The first algorithm to be compared with is NOMAD which is a discrete optimization solver based on mesh adaptive direct search algorithm. Based on our numerous simulations, NOMAD has almost the same performance as other conventional solvers but it is much faster. The other algorithm is the continuous CEP (solving (3) using a quasiNewton algorithm), as an upper bound on the performance.

Since the overall data rate is the final goal, we use the average rate per user as the main comparison criterion. Since the actual sum-rate of CEP is not available in the literature, we use an achievable data rate of CEP developed in [4] as stated in (17) for our numerical examples. In our simulations, we consider Rayleigh flat fading with independent zero mean and unit variance channel coefficients. The number of BS antennas $N$, the number of users $K$, the noise variance $\sigma_{w}^{2}$ and the transmit power $P_{T}$ are set to $100,10,1$ and $10 \mathrm{dBW}$, respectively, unless mentioned otherwise. In discrete-phase algorithms, possible values of the antenna outputs are from a set of $M$-PSK symbols. All the figures are obtained by averaging over 100 realizations of the channel.

As for the choice of the symbol energy, $E_{k}$, the optimal value can be obtained by maximizing (18) over $E_{k}$ 's. However, to circumvent this exhaustive search, we take $E_{k}=P_{T}$, which is a near-optimal choice based on our extensive simulations.

Since solving the continuous CEP is less complex than the discrete CEP, an idea that comes to mind is to solve the continuous CEP problem first, and then, quantize the obtained phases to the desired set of predefined phases. We have tried this approach for many examples, and observed that there is a high total phase error especially for small constellation sizes and large number of antennas. As a result, due to the sensitivity of precoding coefficients to phase errors, the overall performance degrades considerably. For instance, considering above system parameters with 4-ary PSs, the relative achievable rate of the quantized version of the continuous CEP with respect to the proposed TB-CEP with $L=2$ decreases from $87 \%$ for $N=10$ to $51 \%$ for $N=100$. We do not consider this approach any further because of its significantly inferior performance.

Another way of solving discrete optimization problems such as discrete-phase CEP problem in (4) is to utilize semidefinite relaxation (SDR) method [21], [22]. In order to study its effectiveness, we have performed simulations using this technique as well. We have observed that the SDR method outperforms the newly proposed TB-CEP algorithm when there are limited number of BS antennas (i.e., for regular MIMO systems), however, it has a significantly inferior performance in the $\mathrm{m}-\mathrm{MIMO}$ regime. For instance, considering the above system parameters with 4-ary PSs, the relative achievable rate of the SDR method with respect to the proposed TB-CEP with $L=2$ decreases from $120 \%$ for $N=30$ to $54 \%$ for $N=100$.

\section{A. System Performance}

In Fig. 3, the average interference power per user versus the number of BS antennas is shown. It can be seen that the interference powers of all the algorithms decrease as $N$ increases, which is in accordance with Theorem 1. Fig. 3 also gives us the minimum number of base station antennas required for a certain interference level. For instance, the 


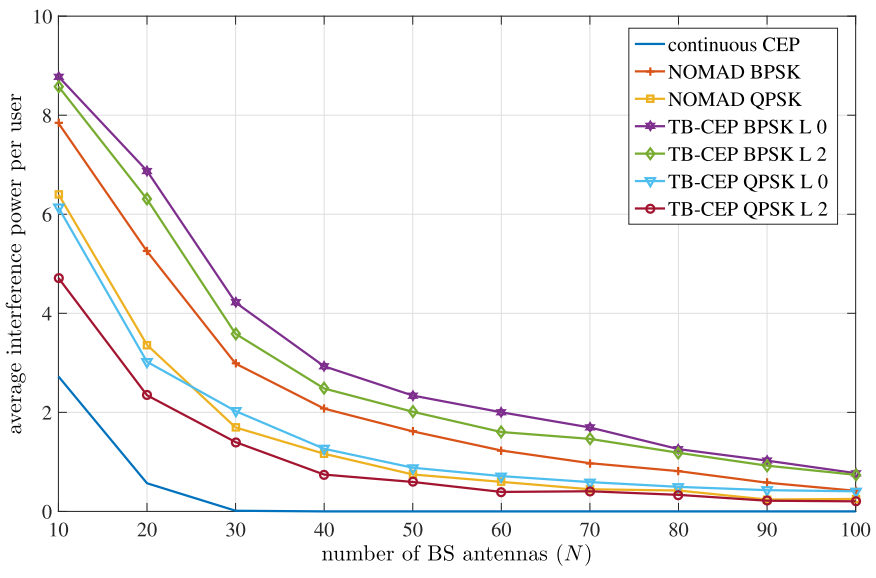

Fig. 3. Average interference power per user versus number of BS antennas $(K=10)$.

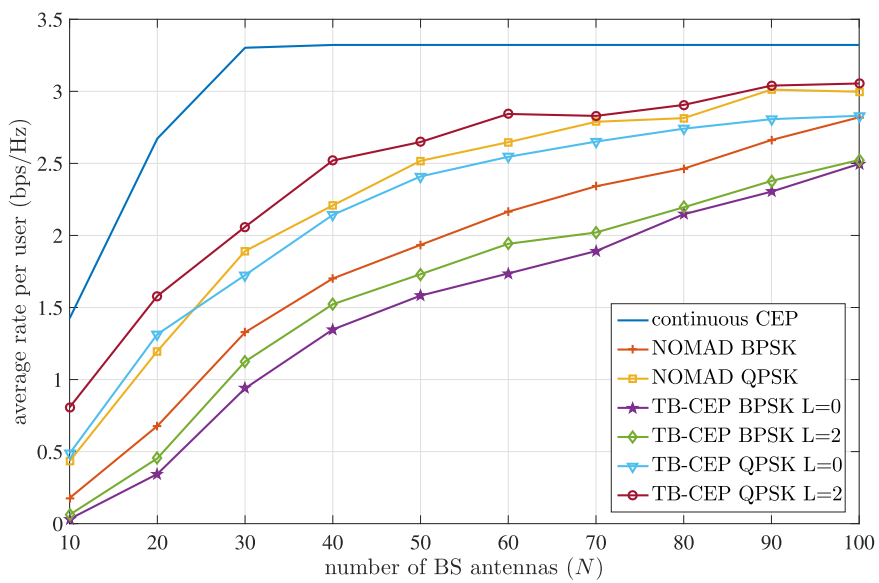

Fig. 4. Average rate per user versus number of BS antennas $(K=10)$.

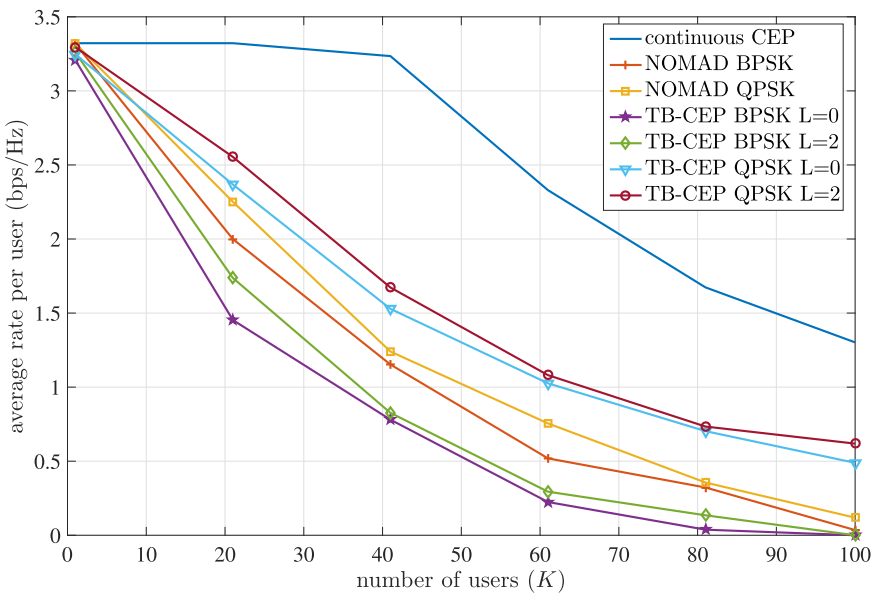

Fig. 5. Average rate per user versus number of users $(N=100)$.

minimum number of $\mathrm{BS}$ antennas required for an average interference power per user of less than one (which is the same value as the noise power considered in the simulations) is 18,36 and 44 for continuous CEP, TB-CEP QPSK with $L=2$ and NOMAD QPSK, respectively.

The average rate per user versus the number of BS antennas is presented in Fig. 4. It can be seen that the rate increases until it saturates for all the algorithms as $N$ increases. According

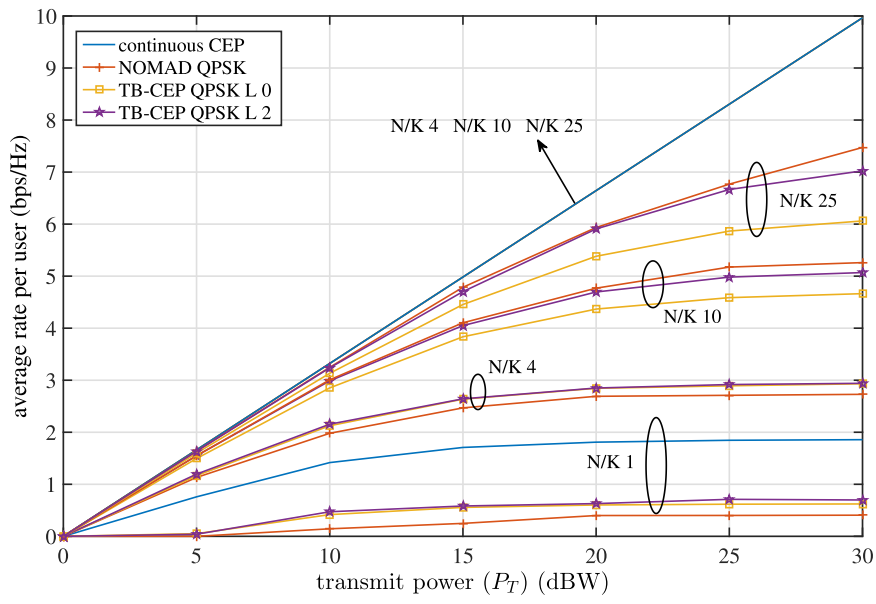

Fig. 6. Average rate per user versus transmit power.

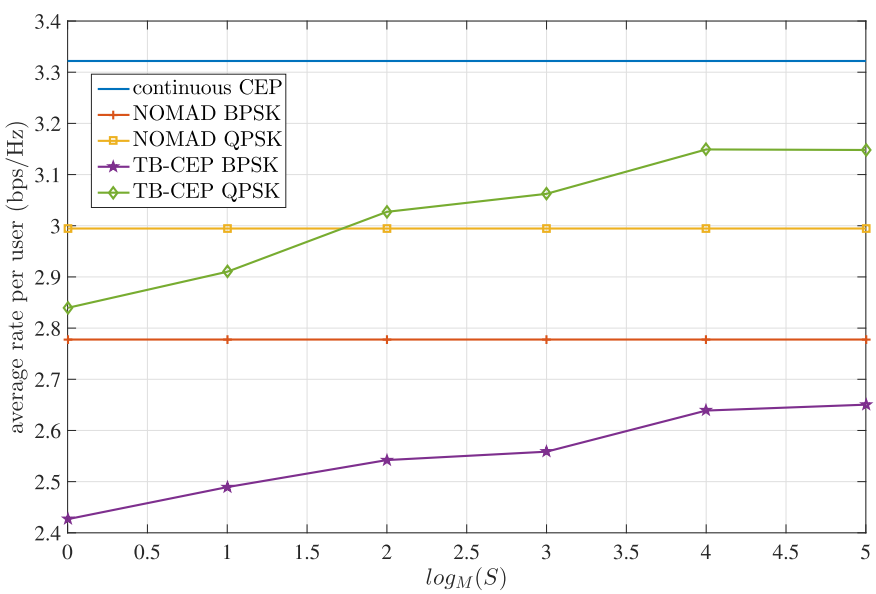

Fig. 7. Average rate per user versus number of trellis states.

to Fig. 4, for higher numbers of BS antennas (in the mMIMO regime), TB-CEP has almost the same performance as the continuous CEP and surpasses NOMAD for the case of 4-ary PSs. For instance, for $N=50$ with 4-ary PSs, TB-CEP with $L=0$ and $L=2$ achieve about $73 \%$ and $80 \%$ of the performance of the continuous CEP, respectively. These percentages increase to about $85 \%$ and $92 \%$ for $N=100$.

The average rate per user versus the number of users $(K)$ is plotted in Fig. 5. As $K$ increases, while $N$ is kept constant, the average rate per user decreases. This is because of the fact that by increasing $K$, the per-user interuser interference increases. As it is shown in Fig. 5, the newly proposed algorithm again offers a significantly better performance than NOMAD.

In Fig. 6, the average rate per user is plotted as a function of the transmit power for $N=100$. We observe that by increasing the transmit power, the performances of all the algorithms improve until they saturate at a certain point. Also, the performances of all the algorithms improve as the $N / K$ ratio (degree of freedom per user) increases. For instance, 4-ary TB-CEP with $L=2$ and $P_{T}=10 \mathrm{dBW}$ for $N / K=1, N / K=4$ and $N / K=10$ achieves about $33 \%, 65 \%$ and $90 \%$ of the rate offered by the continuous CEP, respectively.

In Fig. 7, the average rate per user versus the number of trellis states $\left(S=M^{L}\right)$ is plotted, while keeping the constellation size constant. The performance of the proposed algorithm 


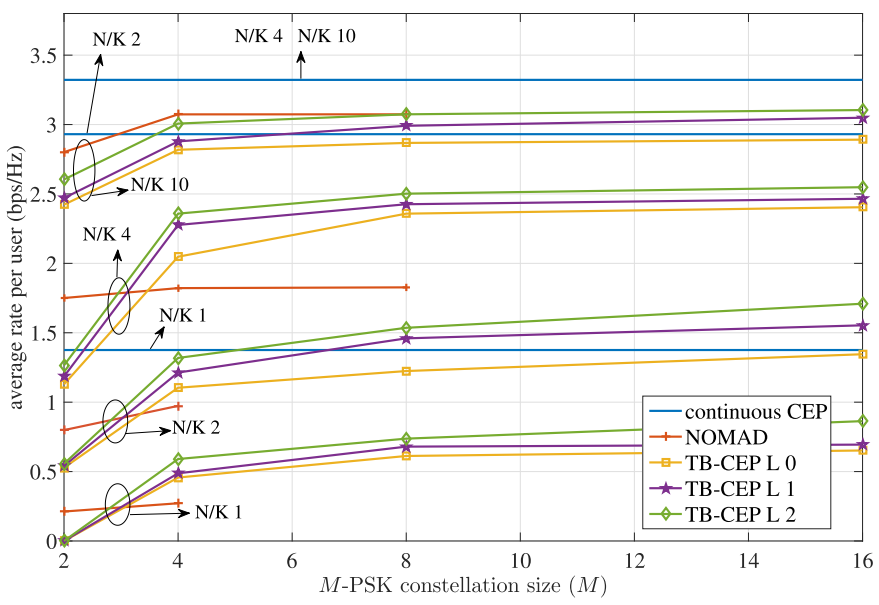

Fig. 8. Average rate per user versus $M$-PSK constellation size.

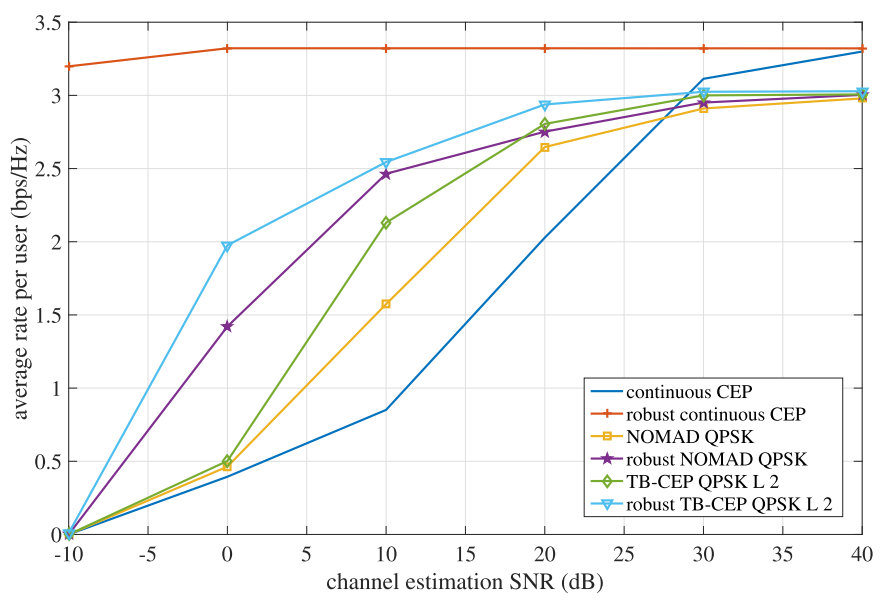

Fig. 9. Average rate per user versus channel estimation SNR.

is improved by increasing the number of states. For instance, in the case of 4-ary PSs, the rate relative to that of continuous CEP increases from $85 \%$ for $L=0$ to $95 \%$ for $L=5$, surpassing that of NOMAD with the same constellation size.

The average rate per user versus the constellation size is presented in Fig. 8 for various $N / K$ ratios for $N=100$. Note that for NOMAD, only the range in which it gives acceptable results is plotted. We observe that, as the constellation size increases, the performances of all the algorithms improve until they rapidly saturate. For instance, for 4-ary PSs with $N / K=10$, TB-CEP with $L=0, L=1$ and $L=2$ achieve about $85 \%, 89 \%$ and $91 \%$ of the rate performance of the continuous CEP, respectively. Also, as expected, we obtain a better performance as $N / K$ increases. For example, 4-ary TB-CEP with $L=2$ achieves about $45 \%, 70 \%$ and $90 \%$ of the rate performance of the continuous CEP for $N / K=2, N / K=4$ and $N / K=10$, respectively.

In Fig. 9, the average rate per user is plotted versus the channel estimation SNR in order to study the effect of errors in channel estimation on the performance of the algorithms. The robust versions of the algorithms are based on the results in Subsection IV.C. We define the channel estimation SNR as $\mathrm{E}\left[\|\mathbf{H}\|_{\mathrm{F}}^{2}\right] / \mathrm{E}\left[\|\tilde{\mathbf{H}}\|_{\mathrm{F}}^{2}\right]$, where $\|\cdot\|_{\mathrm{F}}$ is the Frobenius norm. It is

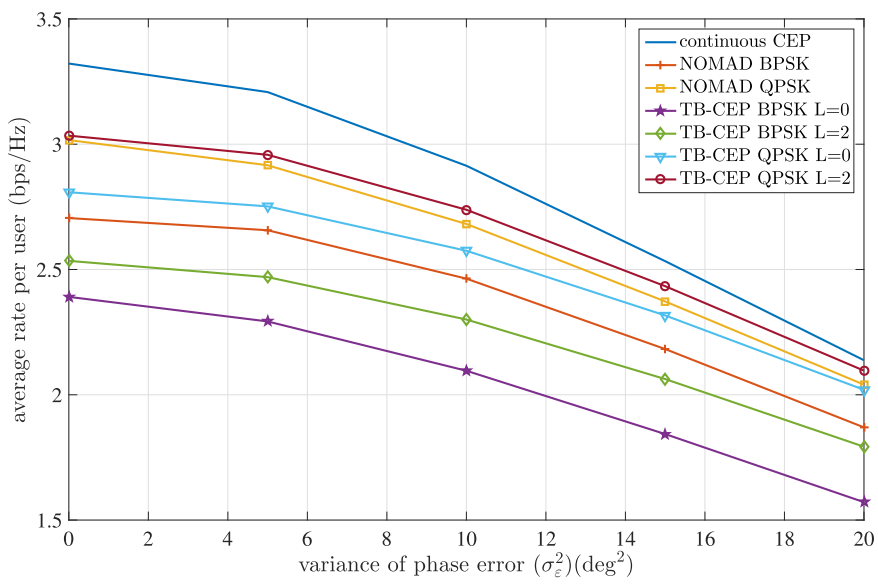

Fig. 10. Average rate per user versus phase error.

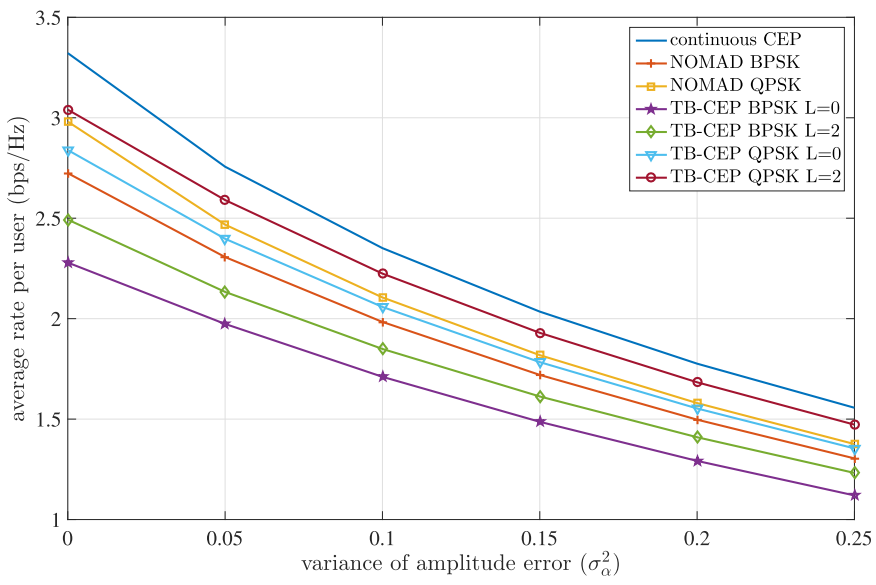

Fig. 11. Average rate per user versus amplitude error.

clear that the performances of all the precoding algorithms improve as the channel estimation SNR increases. The proposed robust TB-CEP has a better performance than TB-CEP especially for moderate values of the channel estimation SNR.

In Fig. 10 and Fig. 11, the average rate per user is plotted against the phase and amplitude error variances in the RF PSs, respectively. We clearly see that while the performance degrades for all the algorithms as the imperfections in the RF PSs increase, the proposed algorithm is fairly robust, in the sense that it shows the same level of degradation as the continuous-phase algorithm of [4].

Finally, as a verification of the result in Theorem 2, the average rate loss per user due to phase and amplitude errors versus SNR is plotted in Fig. 12 for $N=100$. The phase and the amplitude error variances are assumed to be $\sigma_{\varepsilon}^{2}=5 \mathrm{deg}^{2}$ and $\sigma_{\alpha}^{2}=0.1$, respectively. Noting that the expressions in Theorem 2 are obtained under the assumption that a good CEP precoder minimizes the objective function well enough for $K \ll N$ to make the interference power at each user much smaller than the noise power, as it can be seen in Fig. 12, we obtain better approximations of the rate loss due to the phase and amplitude errors for both low and high SNR regimes as $N / K$ increases. 
TABLE I

COMPLEXITY-PERFormance TRADE-OFF OF TB-CEP

\begin{tabular}{|c|c|c|c|c|c|c|c|c|}
\hline & \multicolumn{2}{|c|}{$L=0$} & \multicolumn{2}{c|}{$L=1$} & \multicolumn{2}{c|}{$L=2$} & \multicolumn{2}{c|}{$L=3$} \\
\hline \multirow{2}{*}{$M=2$} & $t_{c}=1 \%$ & $r_{c}=71 \%$ & $t_{c}=80 \%$ & $r_{c}=72 \%$ & $t_{c}=156 \%$ & $r_{c}=76 \%$ & $t_{c}=286 \%$ & $r_{c}=78 \%$ \\
\cline { 2 - 9 } & $t_{d}=0.5 \%$ & $r_{d}=85 \%$ & $t_{d}=39 \%$ & $r_{d}=86 \%$ & $t_{d}=76 \%$ & $r_{d}=90 \%$ & $t_{d}=139 \%$ & $r_{d}=94 \%$ \\
\hline \multirow{2}{*}{$M=4$} & $t_{c}=0.9 \%$ & $r_{c}=86 \%$ & $t_{c}=29 \%$ & $r_{c}=87 \%$ & $t_{c}=1147 \%$ & $r_{c}=91 \%$ & $t_{c}=4644 \%$ & $r_{c}=93 \%$ \\
\cline { 2 - 9 } & $t_{d}=0.003 \%$ & $r_{d}=94 \%$ & $t_{d}=1 \%$ & $r_{d}=95 \%$ & $t_{d}=4 \%$ & $r_{d}=100 \%$ & $t_{d}=17 \%$ & $r_{d}=102 \%$ \\
\hline
\end{tabular}

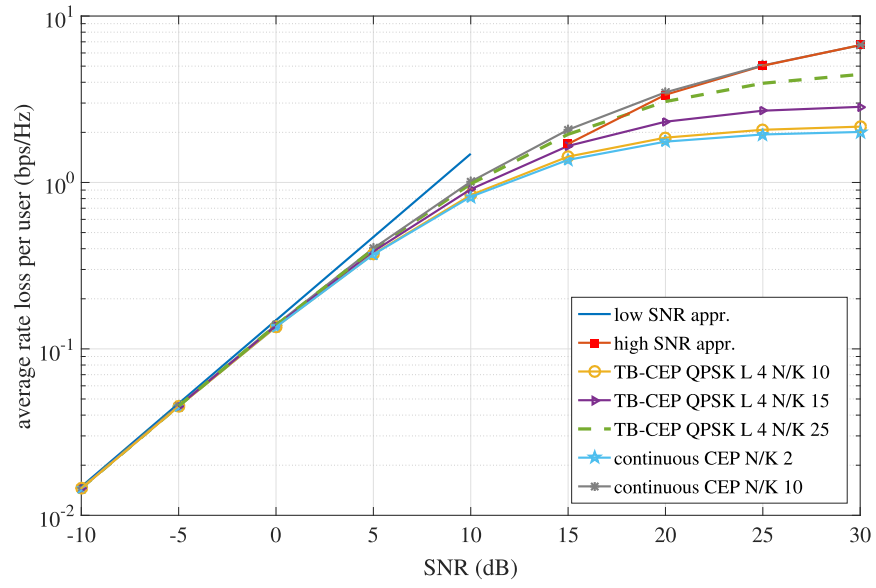

Fig. 12. Average rate loss per user due to phase and amplitude errors versus SNR.

\section{B. Computational Complexity}

The complexity-performance trade-off of the proposed algorithm is presented in Table I, where $t_{c}$ and $t_{d}$ are the relative computation times of TB-CEP with respect to continuous CEP [4] and NOMAD [13], respectively. Also, $r_{c}$ and $r_{d}$ are the relative achievable rates with respect to the continuousphase CEP and NOMAD, respectively.

According to Table I, by increasing the trellis memory (or equivalently, the number of states), the relative performance of the proposed algorithm improves at the cost of increased computational time. This trade-off can help us to select a practical trellis memory as a design parameter. For example, for $M=2$ with $L=0$, we achieve $85 \%$ of the rate of NOMAD, with only $0.5 \%$ of its computation time. For this specific example, the relative computation time increases while the relative performance does not change significantly with increasing $L$, making $L=0$ a good choice for both cases of $M=2$ and $M=4$.

\section{CONCLUSIONS}

In this paper, we have formulated and solved the discretephase CEP problem for m-MIMO systems. We have utilized the sum of interference powers as the objective function and reformulated it as a sum of real terms. Then, by appropriately defining states, we have proposed a low-complexity trellis-based algorithm to solve the reformulated optimization problem. We have also extended the algorithm to be robust against channel estimation errors. Based on extensive numerical results, we conclude that the performance of the proposed algorithm is comparable to the conventional ones (which have exponential complexity), and in some cases it even surpasses them, especially in the m-MIMO scenario. Also, compared to the continuous-phase algorithm, as an upper-bound on performance, the proposed algorithm shows only a small degradation. Furthermore, the performance loss decreases as the number of antennas is increased. We also provide important analytical results. We prove that by increasing the number of BS antennas, user interference can be guaranteed to be arbitrarily small, and we derive closed-form expressions for rate loss due to errors in the phase and the amplitude of the PSs.

\section{APPENDIX}

Knowing that $\varepsilon$ and $\alpha$ are zero mean with variances $\sigma_{\varepsilon}^{2}$ and $\sigma_{\alpha}^{2}$, respectively, the expected value of the interference power in the presence of phase and amplitude errors is

$$
\begin{aligned}
\mathrm{E}_{\varepsilon, \alpha} & {\left[I_{k}(\boldsymbol{\theta}+\boldsymbol{\epsilon}, \boldsymbol{\alpha})\right] } \\
= & \mathrm{E}_{h, x, \varepsilon, \alpha}\left[\frac{P_{T}}{N} \sum_{i=1}^{N}\left(1+\alpha_{i}\right)^{2}\left|h_{k, i}\right|^{2}\right. \\
& +\frac{P_{T}}{N} \sum_{\substack{i=1 \\
N}}^{N} \sum_{\substack{j=1 \\
j \neq i}}^{N} h_{k, i} h_{k, j}^{*}\left(1+\alpha_{i}\right)\left(1+\alpha_{j}\right) e^{j\left(\varepsilon_{i}-\varepsilon_{j}\right)} e^{j\left(\theta_{i}-\theta_{j}\right)} \\
& \left.-2 \sqrt{\frac{P_{T} E_{k}}{N}} \operatorname{Re}\left\{\sum_{i=1}^{N} h_{k, i}\left(1+\alpha_{i}\right) e^{j\left(\theta_{i}+\varepsilon_{i}\right)} x_{k}^{*}\right\}+E_{k}\left|x_{k}\right|^{2}\right] .
\end{aligned}
$$

Since $\mathrm{E}_{\varepsilon, \alpha}[f(\varepsilon, \alpha)]=\mathrm{E}_{\varepsilon}\left[\mathrm{E}_{\alpha \mid \varepsilon}[f(\varepsilon, \alpha)]\right]$, we have

$$
\begin{aligned}
\mathrm{E}_{\varepsilon, \alpha} & {\left[I_{k}(\boldsymbol{\theta}+\boldsymbol{\epsilon}, \boldsymbol{\alpha})\right] } \\
= & \mathrm{E}_{h, x}\left[\varphi_{\mathrm{E}}(-1) \varphi_{\mathrm{E}}(1)\left|\sqrt{\frac{P_{T}}{N}} \mathbf{h}_{k} e^{j \boldsymbol{\theta}}-\sqrt{E_{k}} x_{k}\right|^{2}\right. \\
& +\frac{P_{T}}{N}\left(1+\sigma_{\alpha}^{2}-\varphi_{\mathrm{E}}(-1) \varphi_{\mathrm{E}}(1)\right) \sum_{i=1}^{N}\left|h_{k, i}\right|^{2} \\
& +\left(1-\varphi_{\mathrm{E}}(-1) \varphi_{\mathrm{E}}(1)\right) E_{k}\left|x_{k}\right|^{2} \\
& \left.-2 \sqrt{\frac{P_{T} E_{k}}{N}}\left(\varphi_{\mathrm{E}}(1)-\varphi_{\mathrm{E}}(-1) \varphi_{\mathrm{E}}(1)\right) \operatorname{Re}\left\{\mathbf{h}_{k} e^{j \boldsymbol{\theta}} x_{k}^{*}\right\}\right],
\end{aligned}
$$

where $\varphi_{\mathrm{E}}(t) \triangleq \mathrm{E}_{\varepsilon}\left[e^{j t \varepsilon}\right]$ is the characteristic function of $\varepsilon$. We assume that the phase error is a zero mean symmetric RV, which is a practical assumption. The characteristic function of a zero mean symmetric RV is a real-valued even function [15]. Therefore, assuming the desired transmitted symbols have unit 
power and knowing that channel gains are zero mean with unit variances, and that we have $\sqrt{\frac{P_{T}}{N}} \mathbf{h}_{k} e^{j \boldsymbol{\theta}} \approx \sqrt{E_{k}} x_{k}$ for large number of antennas and $K \ll N$, (33) can be simplified as

$\mathrm{E}_{\varepsilon, \alpha}\left[I_{k}(\boldsymbol{\theta}+\boldsymbol{\epsilon}, \boldsymbol{\alpha})\right] \approx P_{T}\left(\left(1+\rho_{k}\right)+\sigma_{\alpha}^{2}-\left(1+\rho_{k}\right) \varphi_{\mathrm{E}}^{2}(1)\right)$,

where $\rho_{k} \triangleq \frac{E_{k}}{P_{T}}$.

\section{REFERENCES}

[1] T. L. Marzetta, "Noncooperative cellular wireless with unlimited numbers of base station antennas," IEEE Trans. Wireless Commun., vol. 9, no. 11 , pp. $3590-3600$, Nov. 2010.

[2] T. L. Marzetta, "Massive MIMO: An introduction," Bell Labs Tech. J., vol. 20, pp. 11-22, 2015.

[3] E. Larsson, O. Edfors, F. Tufvesson, and T. Marzetta, "Massive MIMO for next generation wireless systems," IEEE Commun. Mag., vol. 52, no. 2, pp. 186-195, Feb. 2014.

[4] S. K. Mohammed and E. G. Larsson, "Per-antenna constant envelope precoding for large multi-user MIMO systems," IEEE Trans. Commun., vol. 61, no. 3, pp. 1059-1071, Mar. 2013.

[5] S. C. Cripps, RF Power Amplifiers for Wireless Communications, 2nd ed. Norwood, MA, USA: Artech House, 2006.

[6] S. K. Mohammed and E. G. Larsson, "Single-user beamforming in largescale MISO systems with per-antenna constant-envelope constraints: The doughnut channel," IEEE Trans. Wireless Commun., vol. 11, no. 11, pp. 3992-4005, Nov. 2012.

[7] S. K. Mohammed and E. G. Larsson, "Constant-envelope multi-user precoding for frequency-selective massive MIMO systems," IEEE Wireless Commun. Lett., vol. 2, no. 5, pp. 547-550, Oct. 2013.

[8] J.-C. Chen, C.-K. Wen, and K.-K. Wong, "Improved constant envelope multiuser precoding for massive MIMO systems," IEEE Commun. Lett., vol. 18, no. 8, pp. 1311-1314, Aug. 2014.

[9] S. Mukherjee and S. K. Mohammed, "Constant-envelope precoding with time-variation constraint on the transmitted phase angles," IEEE Wireless Commun. Lett., vol. 4, no. 2, pp. 221-224, Apr. 2015.

[10] Microwaves101. (Oct. 2015). Phase Shifters. [Online]. Available: http://www.microwaves101.com/encyclopedias/phase-shifters

[11] R. G. Parker and R. L. Rardin, Discrete Optimization. San Diego, CA, USA: Academic, 1988.

[12] V. S. Varadarajan, "A useful convergence theorem," in Proc. Int. Statist. Conf., vol. 20. 1958, pp. 221-222.

[13] S. Le Digabel, "Algorithm 909: NOMAD: Nonlinear optimization with the MADS algorithm," ACM Trans. Math. Softw., vol. 37, no. 4, Feb. 2011, Art. no. 44.

[14] P. Billingsley, Probability and Measure, 4th ed. Hoboken, NJ, USA: Wiley, 2012.

[15] R. M. Dudley, Real Analysis and Probability. Cambridge, U.K.: Cambridge Univ. Press, 2002.

[16] J. E. Mitchell, "Branch-and-cut algorithms for combinatorial optimization problems," in Proc. Handbook Appl. Optim., Jan. 2002, pp. 65-77.

[17] L. Lu, G. Y. Li, A. L. Swindlehurst, A. Ashikhmin, and R. Zhang, "An overview of massive MIMO: Benefits and challenges," IEEE J. Sel. Topics Signal Process., vol. 8, no. 5, pp. 742-758, Oct. 2014.

[18] E. Bjornson, E. G. Larsson, and T. L. Marzetta, "Massive MIMO: Ten myths and one critical question," IEEE Commun. Mag., vol. 54, no. 2, pp. 114-123, Feb. 2016.

[19] S. Boyd and L. Vandenberghe, Convex Optimization. Cambridge, U.K.: Cambridge Univ. Press, 2004.

[20] M. F. Hanif, L. N. Tran, A. Tolli, and M. Juntti, "Computationally efficient robust beamforming for SINR balancing in multicell downlink with applications to large antenna array systems," IEEE Trans. Commun., vol. 62, no. 6, pp. 1908-1920, Jun. 2014.
[21] Z. Q. Luo, W. K. Ma, A. M. C. So, Y. Ye, and S. Zhang, "Semidefinite relaxation of quadratic optimization problems," IEEE Signal Process. Mag., vol. 27, no. 3, pp. 20-34, May 2010.

[22] W.-K. Ma, P.-C. Ching, and Z. Ding, "Semidefinite relaxation based multiuser detection for M-ary PSK multiuser systems," IEEE Trans. Signal Process., vol. 52, no. 10, pp. 2862-2872, Oct. 2004.

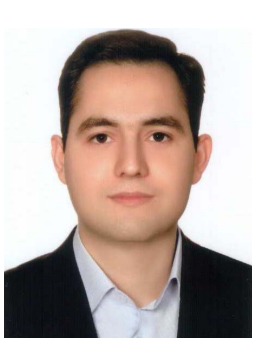

Mohammad Kazemi (S'14) received the B.S. and M.S. degrees in electrical engineering from the Khaje Nasir Toosi University of Technology, Tehran, Iran, in 2007 and 2010, respectively. He is he is currently pursuing the Ph.D. degree with the Electrical Engineering Department, Amirkabir University of Technology, Tehran. He was a Visiting Student with Bilkent University, Turkey, in 2015. His research interests are in wireless communications and signal processing, with particular focus on m-MIMO systems.

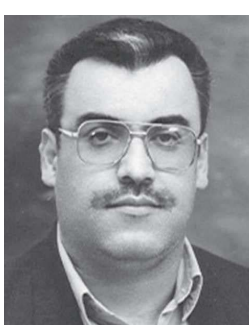

Hassan Aghaeinia received the B.Sc. and M.Sc. degrees from the Amirkabir University of Technology (Tehran Polytechnic), Tehran, Iran, in 1987 and 1989, respectively, and the M.Sc. and Ph.D. degrees from Valenciennes University, Valenciennes, France, in 1992 and 1996, respectively, all in electronic engineering. Since 1996, he has been a Faculty Member of the Amirkabir University of Technology, where he is currently an Associate Professor with the Communication Engineering Group. His research includes work in advanced communication systems and digital image processing.

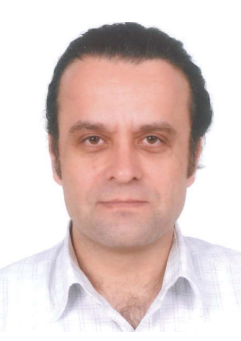

Tolga M. Duman (S'95-M'98-SM'03-F'11) received the B.S. degree from Bilkent University, Ankara, Turkey, in 1993, and the M.S. and Ph.D. degrees from Northeastern University, Boston, MA, USA, in 1995 and 1998, respectively, all in electrical engineering. He was with the Electrical Engineering Department, Arizona State University, as an Assistant Professor from 1998 to 2004, an Associate Professor from 2004 to 2008, and a Professor from 2008 to 2015 . He is currently a Professor of Electrical and Electronics Engineering Department, Bilkent University, and an Adjunct Professor with the School of ECE, Arizona State University. His current research interests are in systems, with particular focus on communication and signal processing, including wireless and mobile communications, coding/modulation, coding for wireless communications, data storage systems, and underwater acoustic communications.

Dr. Duman was a recipient of the National Science Foundation CAREER Award and the IEEE Third Millennium Medal. He served as an Editor of the IEEE TRANSACtion ON Wireless COMMUNiCATIONS from 2003 to 2008, the IEEE COMMUNiCATIONS SURVEYS AND TUTORIALS from 2002 to 2007, the IEEE TRANSACTION ON COMMUNICATIONS from 2007 to 2012, and the Physical Communication (Elsevier) from 2010 to 2016. $\mathrm{He}$ has been the coding and communication theory Area Editor of the IEEE TRANSACTION ON COMMUNICATIONS since 2011, an Editor of the IEEE TRANSACTION ON WiRELESS COMMUNICATION since 2016, and the Editor-in-Chief of the Physical Communication (Elsevier) since 2016. 